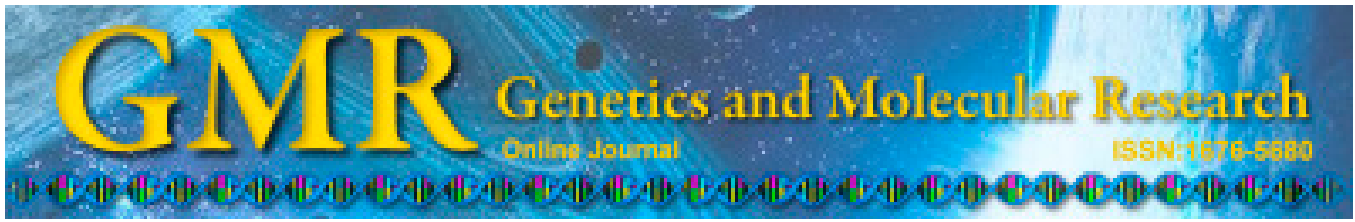

\title{
Ara-C and anti-CD47 antibody combination therapy eliminates acute monocytic leukemia THP-1 cells in vivo and in vitro
}

\author{
Y. Wang, C. Yin, L. Feng, C. Wang and G. Sheng \\ Department of Pediatrics, The First Affiliated Hospital of Zhengzhou University, \\ Zhengzhou, Henan, China \\ Corresponding author: Y. Wang \\ E-mail: yingchaowangcn@yeah.net
}

Genet. Mol. Res. 14 (2): 5630-5641 (2015)

Received August 8, 2014

Accepted January 12, 2015

Published May 25, 2015

DOI http://dx.doi.org/10.4238/2015.May.25.15

\begin{abstract}
Leukemia stem cells (LSCs) are regarded as the origin of leukemia and its recurrence. Side population (SP) cells possess some intrinsic stem cell properties and contain numerous LSCs. In this study, we examined the prognostic significance of cluster differentiation 47 (CD47) and identified the appropriate target for eliminating LSCs. We determined the percentage of SP cells in a THP-1 cell population and analyzed CD47 expression in different cell subsets. We then explored whether CD47 affected the phagocytic ability of macrophages to LSCs in vitro. Finally, the effect of anti-CD47 monoclonal antibodies, alone or combination with cytarabine, against leukemic cells was evaluated in vitro and in vivo to identify the optimal targets for the treatment of leukemia. We observed an SP sub-fraction at low frequency (1.81 $\pm 0.99 \%$ ), which was a likely candidate for LSC enrichment. CD47 was more highly expressed on THP-1 LSCs $(\mathrm{P}<0.05)$ and was an independent predictor of survival and refractory disease in THP-1engrafted mice. Furthermore, the anti-CD47 monoclonal antibody stimulated preferential phagocytosis of LSCs by macrophages in vitro. Finally, single or combination treatment of THP-1 LSC-engrafted mice
\end{abstract}


with cytarabine and anti-CD47 antibody resulted in targeting of LSCs and depletion of leukemia cells. These findings suggest that CD47 is an antibody target in LSCs and combination treatment with cytarabine and anti-CD47 monoclonal antibody represents an attractive option for the therapeutic targeting of acute monocytic leukemia.

Key words: Acute monocytic leukemia; CD47; Cytarabine; Leukemia stem cells; Monoclonal antibody

\section{INTRODUCTION}

Childhood and adolescent acute myeloid leukemia (AML) is traditionally one of the most difficult childhood cancers to treat successfully (Woods, 2006). The relapse rate remains unacceptably high, with a 5-year event-free survival of approximately 50\% (Gorman et al., 2010). Furthermore, this can only be achieved using highly intensive chemotherapy, which results in relatively high rates of treatment-related deaths and significant late effects (Kaspers and Zwaan, 2007). Although much effort is being focused on improving these limitations, an efficient and effective method has not been developed. Therefore, novel therapeutic strategies are urgently required to improve the unsatisfactory prognosis of this disease.

In various leukemias, only small subpopulations of cells have the potential to transfer disease upon transplantation into immunocompromised non-obese diabetic/severe combined immunodeficiency (NOD/SCID) mice, and markers that distinguish the leukemogenic cancer cells from the bulk population of non-leukemogenic cells have been identified (Testa, 2011). If only a rare subset of leukemia stem cells (LSCs) drives leukemic formation, the goal should be to identify and develop therapies that target this population. Side population (SP) cells are defined by Hoechst 33342 exclusion and can be detected by flow cytometry. These cells represent only a small fraction of the total population, and thus investigation of the properties of SP cells represents an important research focus (Goodell et al., 1996; Zhou et al., 2002; Hussain et al., 2005). SP analysis can be used to identify cancer stem cell populations (Haraguchi et al., 2006; Chiba et al., 2006), and these cells share the largest number of relevant features of LSCs, including self-renewal potential and quiescent status (Setoguchi et al., 2004). SP cell sorting using flow cytometry is a convenient method for isolating LSCs (Guo et al., 2003). Emerging evidence has demonstrated that monoclonal antibodies, either alone or in combination, are an effective modality for cancer treatment (Adams and Weiner, 2005). Cluster differentiation 47 (CD47) serves as the ligand for signal regulatory protein alpha (SIRP $\alpha$ ), which is expressed on phagocytic cells, including macrophages and dendritic cells, and when activated initiates a signal transduction cascade resulting in inhibition of phagocytosis (Barclay and Brown, 2006). Therefore, anti-CD47 monoclonal antibodies may represent a suitable therapy for targeting LSCs. The THP-1 cell line, which was originally established from an infant diagnosed with AML (Tsuchiya et al., 1980), provides an experimental model for the functional analysis of preclinical therapeutics and target identification studies of AML. Cytarabine (Ara-C) is commonly used for the treatment of acute leukemia; incorporation of Ara-C into the DNA is a critical event in the process leading to the death of proliferating leukemic cells, although this is relatively ineffective against LSCs, which are maintained in a quiescent state (Misaghian et al., 2009). In this study, Ara-C was used to kill common proliferating THP-1 cells. In addition, 
anti-CD47 monoclonal antibody therapy targeting THP-1 LSCs was evaluated as an approach to eradicate leukemia cells in vitro and in vivo.

The cancer stem cell theory suggests that chemoresistance and recurrence of tumors often results from the similarity between the stem cell properties of normal and cancer cells (Kayo et al., 2007). To date, AML LSCs are the most well characterized cancer stem cell population (Wang and Dick, 2005). LSCs, like their normal hematopoietic stem cell (HSC) counterparts, possess a range of characteristics that enable their long-term survival, and some facilitate their escape from the cytotoxic effects of chemotherapy. First, LSCs exist primarily in a quiescent phase of the cell cycle (Guan et al., 2003). Second, by reducing cytotoxic stress, LSCs represent a potential reservoir for selecting mutants that are resistant to targeted or conventional therapy (Heidel et al., 2006). LSCs can undergo mutations and epigenetic changes, leading to drug resistance and relapse. Recent studies suggest that mature leukemia cells acquire LSC characteristics, thereby evading chemotherapeutic treatment and sustaining the disease (Ravandi and Estrov, 2006). Third, primitive LSCs possess natural mechanisms of survival, such as drug-efflux capabilities (such as multidrug resistance 1) (Jordan and Guzman, 2004). The use of traditional drugs such as a combination of Ara-C and daunomycin results in relatively high rates of treatment-related deaths and significant late effects, and does not address long-term survival rates (Inaba et al., 2008).

\section{MATERIAL AND METHODS}

\section{Cell line and culture}

The human acute monocytic leukemia cell line THP-1 (Shanghai Institute of Cell Biology, Shanghai, China) was cultured in RPMI 1640 medium (Hyclone, Logan, UT, USA) supplemented with $10 \%$ fetal bovine serum (Hyclone) and $100 \mathrm{U} / \mathrm{mL}$ penicillin-streptomycin (Invitrogen Life Technologies, Carlsbad, CA, USA) at $37^{\circ} \mathrm{C}$ in an atmosphere containing $5 \% \mathrm{CO}_{2}$.

\section{SP cell analysis and fluorescence-activated cell sorting}

Cells were suspended at $1 \times 10^{6}$ cells $/ \mathrm{mL}$ in Hanks' balanced salt solution supplemented with $3 \%$ fetal calf serum and $10 \mathrm{mM}$ HEPES. Cells were then incubated at $37^{\circ} \mathrm{C}$ for $90 \mathrm{~min}$ with $5 \mu \mathrm{g} / \mathrm{mL}$ Hoechst 33342 (Sigma, St. Louis, MO, USA), either alone or in the presence of $50 \mu \mathrm{M}$ verapamil (Sigma). After incubation, $1 \mu \mathrm{g} / \mathrm{mL}$ propidium iodide (BD Biosciences, Franklin Lakes, NJ, USA) was added and cells were then filtered through a $40-\mu \mathrm{m}$ cell strainer (BD) to obtain single-suspension cells. Cell analysis and purification were performed using a MoFlo carrying a triple-laser (DakoCytomation, Glostrup, Denmark). Hoechst 33342 was excited with an ultraviolet laser at $350 \mathrm{~nm}$ and fluorescence emission was measured with 405/ BP30 (Hoechst blue) and 570/BP20 (Hoechst red) optical filters. Propidium iodide labeling was measured through the 630/BP30 filter to discriminate dead cells. SP cells (Komuro et al., 2007) and non-SP cells were isolated from each well.

\section{In vitro phagocytosis assays}

THP-1 SP cells were incubated with either mouse or human macrophages in the presence of $7 \mu \mathrm{g} / \mathrm{mL}$ IgG1 isotype control antibody, anti-CD45 IgG1, or anti-CD47 (eBioscience, 
San Diego, CA, USA) for $2 \mathrm{~h}$. Cells were then analyzed by microscopy to determine the phagocytic index (number of cells ingested per 100 macrophages).

\section{Establishment of THP-1 SP cell xenograft leukemia NOD/SCID mouse model}

All animal studies were carried out in compliance with the Guidelines for the Care and Use of Laboratory Animals in Henan Province, China. Male NOD/SCID mice (aged 4-5 weeks) were obtained from HFK BIOSCIENCE Co. (Beijing, China) and maintained ( $\mathrm{N}=5$ per cage) under specific pathogen-free conditions. Approximately $1-2.5 \times 10^{5}$ fluorescence-activated cell sorting-purified THP-1 SP cells were resuspended in 20-40 $\mu \mathrm{L}$ phosphate-buffered saline and transplanted intravenously via the tail vein. Three to four weeks later, human AML engraftment ( $\mathrm{hCD} 45^{+} \mathrm{CD} 33^{+}$cells) was assessed in the peripheral blood and bone marrow by tail bleed and aspiration of the femur, respectively. Engrafted mice were then treated daily with intraperitoneal injections of $100 \mu \mathrm{g}$ anti-CD47 antibody or IgG (eBioscience) control for 14 days. On day 15, mice were sacrificed and the peripheral blood and bone marrow were analyzed for AML.

\section{Enrichment of LSC subpopulation in the THP-1 human leukemic cell line with Ara-C}

Next, $1 \times 10^{6}$ cells $/ \mathrm{mL}$ THP-1 cells were incubated with $100 \mu \mathrm{g} / \mathrm{mL}$ Ara-C for 24 or $48 \mathrm{~h}$ at $37^{\circ} \mathrm{C}$ in an atmosphere containing $5 \% \mathrm{CO}_{2}$. Cells were then harvested at different time points, washed twice with phosphate-buffered saline, and analyzed for CD47 expression.

\section{Flow cytometric analysis of CD47 expression}

First, CD47 expression was examined in THP-1 SP cells and bulk THP-1 (non-SP) cells sorted by flow cytometry. Next, CD47 expression was examined on normal peripheral blood mononuclear cells (MNCs) and bone marrow MNCs of NOD/SCID mice. Peripheral blood MNCs of leukemia-bearing NOD/SCID mice were also examined. Leukemia-bearing mice were divided into 2 groups according to different levels of CD47 expression (CD47 low and $\mathrm{CD} 47^{\text {high }}$ ) and survival time was monitored. Moribund mice were sacrificed and the peripheral blood, bone marrow, and organs, including the liver and spleen, were analyzed for AML. Expression of CD47 was analyzed using an anti-human CD47 fluorescein isothiocyanate antibody (eBioscience).

\section{In vivo antibody treatment of THP-1 LSC-engrafted mice}

Engrafted mice were randomly divided into 4 groups: IgG1 control, anti-CD47 antibody, Ara-C, and anti-CD47 antibody + Ara-C. Next, $100 \mu \mathrm{g} /$ mouse intraperitoneal injections IgG1 control or anti-CD47 antibody were administered daily for 2 weeks, or $100 \mathrm{mg} \cdot \mathrm{kg}^{-1} \cdot \mathrm{day}^{-1}$ Ara-C for 1 week plus $100 \mu \mathrm{g}$ anti-CD47 antibody for 2 weeks. Treatment was then stopped and mice were monitored for survival analysis. Complete remission was defined as no evidence of leukemia detected by Wright's staining of bone marrow smears and by hematoxylin and eosin staining of pathological sections at the end of treatment. Relapse was defined as evidence of lymphoma detected by Wright's staining of bone marrow smears after the end of treatment in mice with a prior complete remission. 


\section{Statistical analysis}

Statistical analysis was performed using SPSS 17.0 (SPSS, Inc., Chicago, IL, USA). All experiments were repeated 3 or 5 times. Data are reported as means \pm standard deviation. The significance of the differences in mean values among groups was evaluated by using the Student $t$-tests, Kaplan-Meier survival curves with log-rank tests, or single-factor analysis of variance and post-hoc tests. $\mathrm{P}<0.05$ was considered to be statistically significant.

\section{RESULTS}

\section{Prevalence of SP cells in THP-1 cells}

Hoechst 33342 dim cells (located on the bottom left-hand corner of the flow cytometry plot) were gated as the SP population and represented a percentage of $1.81 \pm 0.99 \%$ of the total THP-1 population (Figure 1A). The Hoechst 33342 dim THP-1 SP cells were effectively abolished following staining in the presence of the calcium channel blocker, verapamil (Figure 1B). The SP and non-SP cells in THP-1 cells were sorted separately and purity analysis showed that the sorted SP cells were concentrated in the SP region of the flow cytometry plot. The sorted non-SP cells remained concentrated in the sub-region of the main group of cells identified prior to sorting.
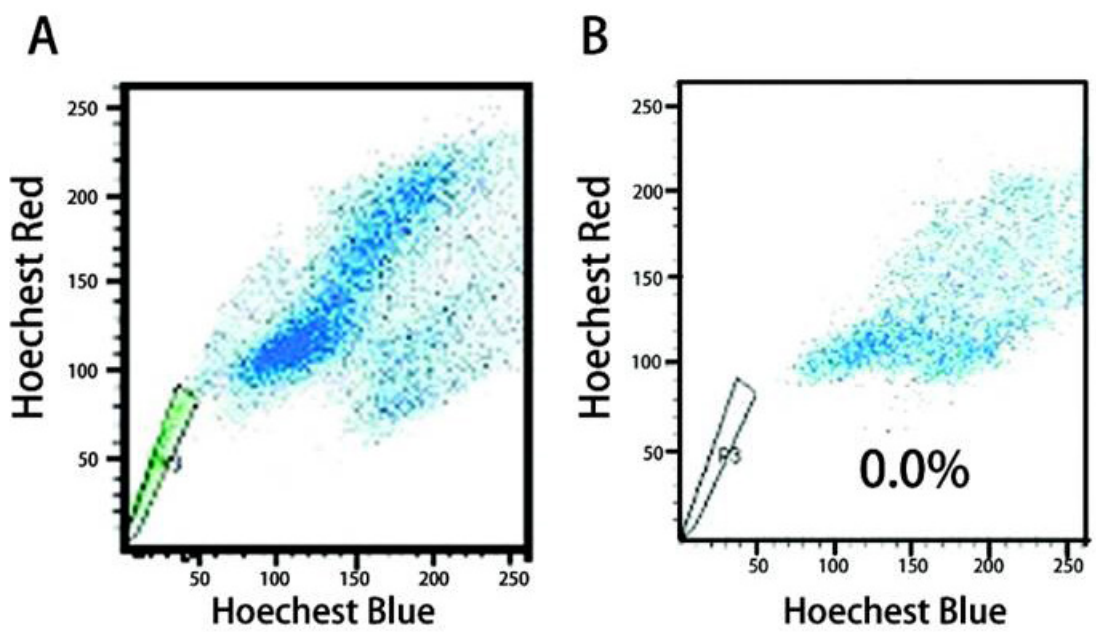

Figure 1. Analysis of SP cells by flow cytometry. A. SP cells appeared as the Hoechst dim fraction capable of Hoechst 33342 exclusion detected by flow cytometry and typically represented $1.81 \pm 0.99 \%$ of viable cells from the THP-1 leukemic cell line. The non-SP cells that retained high levels of Hoechst staining were also referred to as the bulk population cells. B. SP cells were ablated when verapamil was included in the Hoechst 33342 incubation. Verapamil blocks the activity of drug transport proteins, preventing efflux of the dye.

\section{CD47 was more highly expressed on THP-1 LSCs}

Compared with normal MNCs derived from both peripheral blood (NPB) and normal bone marrow (NBM), CD47 was more highly expressed on THP-1 LSCs according to flow cy- 
tometry. Normalized mean expression of CD47 for each population was: $14.1 \pm 3.54 \%$ NPB, $30.7 \pm 1.90 \%$ NBM, $56.3 \pm 9.54 \%$ bulk THP-1, and $73.8 \pm 13.38 \%$ THP-1 LSCs. Statistically significant differences were identified between the mean CD47 expression of NPB compared with that of NBM $(\mathrm{P}=0.000)$, NBM compared with bulk THP-1 cells $(\mathrm{P}=0.000)$, NPB compared with THP-1 LSCs $(\mathrm{P}=0.000)$, bulk THP-1 compared with THP-1 LSCs $(\mathrm{P}=0.002)$, and NPB compared with bulk THP-1 $(\mathrm{P}=0.000)$. Differences in the levels of CD47 expression were detected between cell subgroups. Normalized mean CD47 expression at 24 and $48 \mathrm{~h}$ was $62.1 \pm 9.09 \%$ at $24 \mathrm{~h}$ and $68.5 \pm 8.18 \%$ at $48 \mathrm{~h}$. The differences between the mean CD47 expression levels in these 4 groups $(\mathrm{P}=0.008)$ were statistically significant.

\section{Identification of human THP-1 LSCs xenotransplant leukemia models}

Three to four weeks after intravenous transplantation of THP-1 LSCs via the tail vein, human $\mathrm{AML}$ engraftment of $\mathrm{hCD} 45^{+} \mathrm{CD} 33^{+}$cells was assessed in the peripheral blood and bone marrow by tail bleed and aspiration of the femur, respectively. The results revealed successful establishment of the systemically disseminated leukemia model in all NOD/SCID mice. Wright's staining of bone marrow and peripheral blood smears revealed successful xenotransplantation of leukemia in nearly all mice.

\section{Increased CD47 expression was correlated with poor clinical prognosis and adverse pathology features in human THP-1 LSC-engrafted mice}

Leukemia-bearing mice $(\mathrm{N}=20)$ were divided into 2 groups according to different levels of CD47 expression (median): CD47 low expression group (CD47 low, CD47 expression $<30 \%)$, and CD47 high expression group (CD47 $7^{\text {high }}$, CD47 expression $\left.>30 \%\right)(\mathrm{N}=10$ per group). The prognostic influence of CD47 expression on the overall survival of human THP-1 LSC-engrafted mice is shown in Figure 2. Higher CD47 expression was also associated with poor clinical outcome. In CD47 high mice, the peripheral blood, bone marrow, and organs such as the liver and spleen were packed with monomorphic leukemic blasts, while no leukemia cells were detected in organs such as the liver and spleen in CD47 low mice.

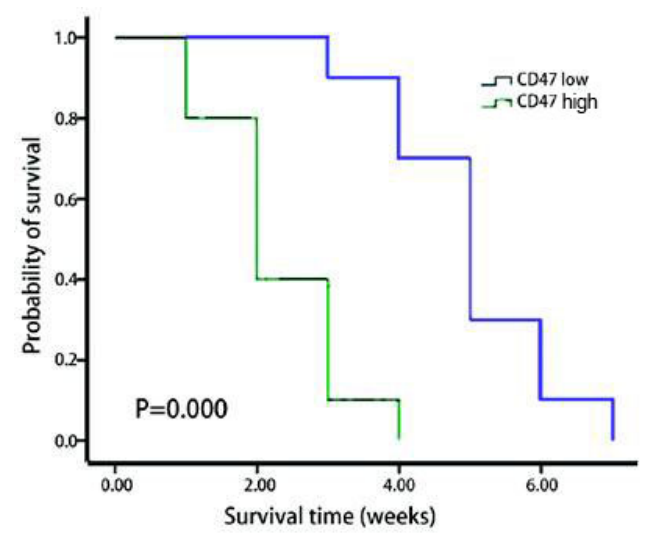

Figure 2. Kaplan-Meier survival curve with log-rank testing. Statistically significant differences were detected between $\mathrm{CD} 47^{\text {high }}$ and $\mathrm{CD} 47^{\text {low }}$ mice $(\mathrm{P}<0.001)$. 


\section{Anti-CD47 antibodies stimulate phagocytosis of LSCs by human macrophages in vitro}

The capacity of anti-human CD47 antibodies to stimulate phagocytosis of human THP-1 LSCs by human peripheral blood macrophages was investigated in vitro. Incubation of THP-1 LSCs in the presence of an IgG1 isotype control or anti-CD45 IgG1 antibody did not result in significant phagocytosis. However, anti-CD47 antibodies stimulated the phagocytosis of THP-1 LSCs. The phagocytic index was determined for each condition by calculating the number of ingested cells per 100 macrophages. For THP-1 LSC, a statistically significant difference was detected between blocking anti-CD47 antibody treatment and treatment with the isotype and anti-CD45 control antibodies $(\mathrm{P}<0.001)$.

\section{Anti-CD47 antibodies stimulate phagocytosis of LSCs by mouse macrophages in vitro}

The capacity of anti-human CD47 antibodies to stimulate phagocytosis of human THP-1 LSC by mouse abdominal cavity-derived macrophages was also evaluated in vitro. Incubation of THP-1 LSCs in the presence of an IgG1 isotype control or anti-CD45 IgG1 antibody did not result in significant phagocytosis. However, anti-CD47 antibodies stimulated the phagocytosis of THP-1 LSCs by mouse macrophages (Figure 3A). The phagocytic index was determined for each condition by calculating the number of ingested cells per 100 macrophages. For THP-1 LSCs, a statistically significant difference was detected between blocking anti-CD47 antibody treatment and treatment with the isotype and anti-CD45 control antibodies $(\mathrm{P}<0.001)$ (Figure 3B).

\section{A}
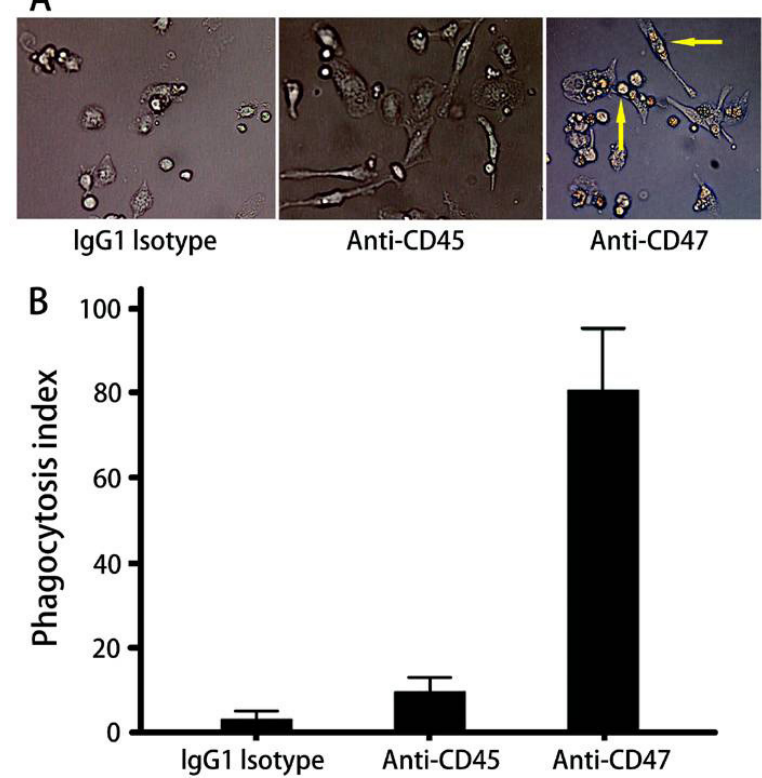

Figure 3. Blocking anti-CD47 antibodies stimulated phagocytosis of LSCs by mouse macrophages in vitro. A. Phagocytosis of THP-1 LSCs by mouse macrophages in vitro. THP-1 LSCs were incubated with mouse macrophages in the presence of the indicated antibodies and then assessed for phagocytosis by microscopy. $\mathbf{B}$. Phagocytosis of THP-1 LSCs by mouse macrophages in vitro. A statistically significant difference was detected between blocking anti-CD47 antibody treatment and treatment with the isotype and anti-CD45 control antibodies $(\mathrm{P}<0.001)$. 


\section{Combination therapy with anti-CD47 antibody and Ara-C eliminates leukemia cells in human THP-1 xenotransplant models}

Human THP-1 xenotransplant mice $(\mathrm{N}=40)$ were randomly divided into 4 groups and were administered daily injections of either the control mouse IgG, anti-CD47 antibody, Ara-C, or anti-CD47 antibody + Ara-C. Anti-CD47 antibody treatment decreased the leukemic burden in the peripheral blood and bone marrow in these mice (Figures 4-5) and significantly prolonged survival compared with control IgG, although all mice eventually died (Figure 6). Similar results were observed following Ara-C treatment and were not statistically different from those detected following anti-CD47 antibody treatment. In contrast, combination therapy with anti-CD47 antibody and Ara-C eliminated leukemia as indicated by long-term survival $(\mathrm{P}<0.001)$.

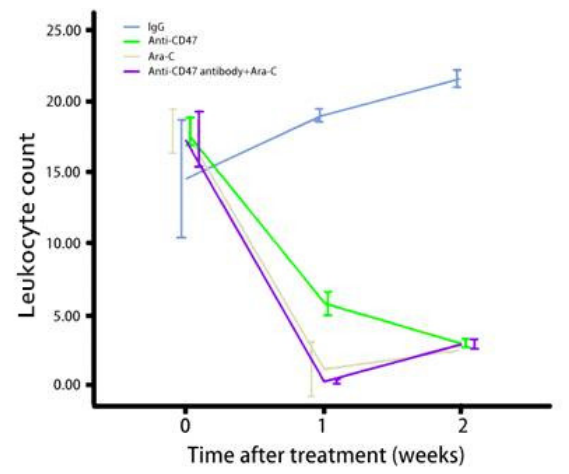

Figure 4. Leukocyte counts in peripheral blood of human THP-1 AML mice. Peripheral blood leucocyte counts of mice treated with control IgG increased gradually, while leukocyte counts of mice treated with anti-CD47 antibody, Ara-C, and anti-CD47 antibody + Ara-C showed marked reduction in leukemia.

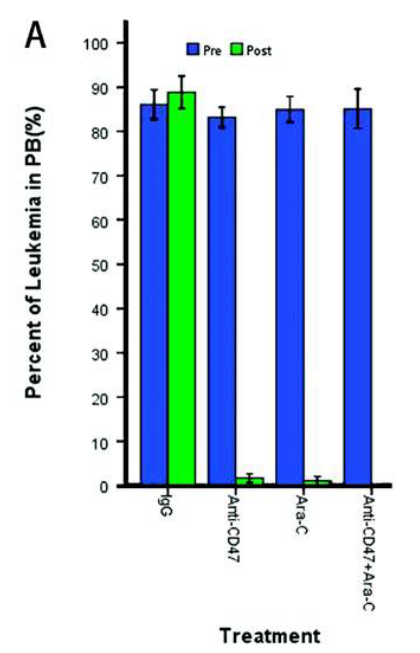

B

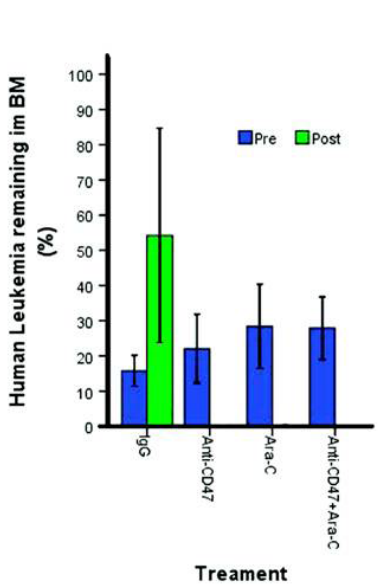

Figure 5. Percentage of leukemia cells in peripheral blood (PB) and bone marrow (BM) of AML mice pre- and posttreatment. A. Percentage of leukemia cells in PB of mice engrafted with THP-1 LSC pre- and post-treatment with either control IgG, anti-CD47, Ara-C, or anti-CD47 antibody + Ara-C. B. Percentage of leukemia cells in BM of mice engrafted with THP-1 LSC pre- and post-treatment with either control IgG, anti-CD47, Ara-C, or anti-CD47 antibody + Ara-C. 


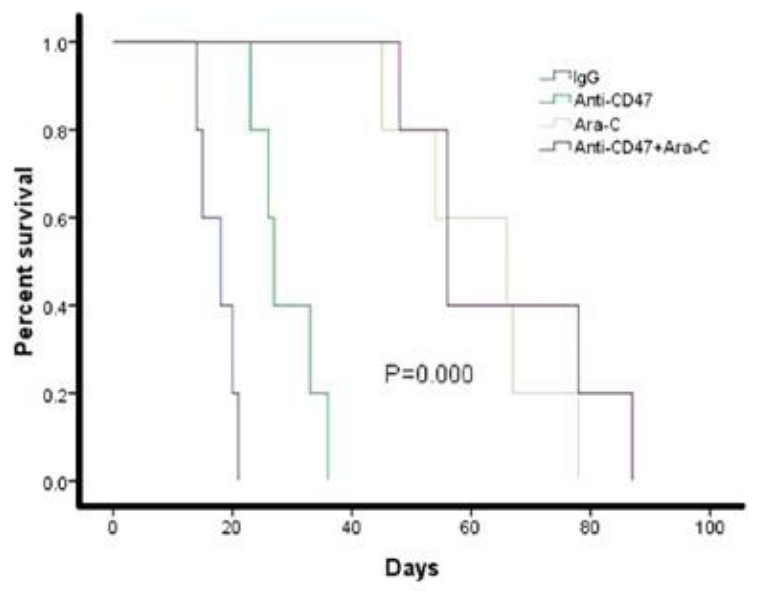

Figure 6. Kaplan-Meier survival analysis of different treatment groups. Kaplan-Meier survival analysis was performed. Anti-CD47 antibody alone or Ara-C + anti-CD47 antibody combination therapy significantly prolonged survival compared with control IgG, although all mice eventually died.

\section{DISCUSSION}

The lack of durable responses in a high percentage of AML patients suggests that current treatments do not effectively target LSCs, which are regarded as the origin of leukemia, and its recurrence after seemingly successful therapy. Elimination of LSCs is required to eradicate leukemia and cure the patient. In this study, the pediatric AML cell line THP-1, derived originally from an infant diagnosed with AML, was used as an experimental model of this disease. A rare SP sub-fraction was identified in the THP-1 cells that were likely enriched for LSCs.

Recent efforts have focused on the development of targeted therapies for LSCs, including monoclonal antibodies (Majeti et al., 2009; Chao et al., 2010). Monoclonal antibodies targeting the specific epitopes expressed on LSCs rather than on HSCs appear to be useful for targeting LSCs in vivo. A previous study found encouraging results in preclinical models, thus supporting the development of these agents (Lane and Gilliland, 2010). Identification of a cell-surface phenotype that distinguishes between LSCs and normal HSCs is essential for the development of prospective separation strategies for use in targeting therapies. Several candidate molecules have recently been identified, including CD123 (Jordan et al., 2000), CD44 (Jin et al., 2006), CD96 (Hosen et al., 2007), and C-type lectin-like molecule-1 (van Rhenen et al., 2007).

CD47 (also known as integrin-associated protein) is a member of the Ig superfamily and contains a single extracellular Ig domain followed by 5 transmembrane segments. This molecule is ubiquitously expressed on most cells and interacts functionally with integrins (Lindberg et al., 1993; Matozaki et al., 2009). Furthermore, CD47 inhibits phagocytosis and as such represents a suitable molecule for antibody-mediated targeting of AML LSCs. CD47 upregulation is an important mechanism that provides protection to normal HSCs during inflammation-mediated mobilization. Leukemic progenitors co-opt this ability in order to evade macrophage phagocytosis (Jaiswal et al., 2009). The absence of CD47 expression on cells results in their phagocytosis; therefore, CD47 acts as a protective signal through the interaction with its receptor, $\operatorname{SIRP} \alpha$, which is expressed by phagocytes. CD47 is also capable 
of interacting with its receptor SRP $\alpha$ (Jiang et al., 1999) on macrophages to negatively regulate phagocytosis (Brown and Frazier, 2001). CD47 expression appears to be an important mechanism by which LSCs evade the host innate immune response. Furthermore, blockade of CD47 with a specific monoclonal antibody leads to increased phagocytosis and reduced engraftment of LSCs with concomitant reduction in leukemic burden (Majeti et al., 2009). Finally, CD47 was found to be more highly expressed on AML LSCs compared with their normal counterparts (Jaiswal et al., 2009) and human non-Hodgkin lymphoma cells (Chao et al., 2010). In the present study, varying CD47 expression was detected within different cell subgroups. Compared to both normal peripheral blood, bone marrow MNCs, and bulk THP1, CD47 was more highly expressed on THP-1 LSCs, which is consistent with the results of previous studies. More importantly, the proportion of CD47-positive cells in each enriched subpopulation was found to vary. CD47 has been reported to be highly expressed on a subset of human acute lymphoblastic leukemia (Chao et al., 2011), AML (Majeti et al., 2009), and non-Hodgkins lymphoma patient samples (Chao et al., 2010). These studies suggest that higher CD47 expression is an independent predictor of adverse clinical outcomes in hematopoietic system cancers. Furthermore, NOD/SCID mouse xenograft transplantation assays of patient AML samples revealed poorer prognosis in these patients (Monaco et al., 2004). In this study, the survival time of $\mathrm{CD} 47^{\text {low }}$ mice was clearly prolonged compared with that of $\mathrm{CD} 47^{\text {high }}$ mice. To investigate leukemic cell infiltration, moribund mice were sacrificed and representative hematoxylin and eosin-stained sections showed that more complete leukemia dissemination was present in CD47 high mice. This indicated that a higher level of CD47 expression is an independent predictor of reduced survival time in human AML LSC-engrafted mice.

CD47-mediated activation of SIRP $\alpha$ initiates a signal transduction cascade resulting in the inhibition of phagocytosis, and treatment with anti-CD47 antibodies not only reduces primary tumor size but also can be used to inhibit the development of, or to eliminate, metastatic disease (Edris et al, 2012). In our study, Ara-C plus anti-CD47 antibody treatment effectively decreased the leukemic burden in these mice and significantly prolonged survival compared with mice treated with control IgG, Ara-C, or anti-CD47 antibody alone. This combination therapy offers several advantages compared with Ara-C and daunomycin combination therapy or monotherapies in AML. First, a combination of Ara-C and daunomycin results in relatively high rates of treatment-related deaths and significant late effects, and does not address long-term survival rates. Therapy with only monoclonal antibodies targeting cancerspecific antigens can result in decreased off-target toxicity compared with current therapeutic regimens that utilize chemotherapy (Chao et al., 2010). Second, Ara-C eliminates leukemia cells during the proliferative phase, while anti-CD47 monoclonal antibodies lead to increased phagocytosis of quiescent-stage leukemia cells. Third, Ara-C eliminates common leukemia cells, while anti-CD47 monoclonal antibody therapy targets THP-1 LSCs, resulting in increased therapeutic efficacy.

In conclusion, the proportion of SP cells in AML THP-1 cells was initially assessed and the optimal approach for eliminating LSCs was investigated for the potential to cure leukemia patients. CD47 was more highly expressed on THP-1 LSCs. Anti-CD47 monoclonal antibody therapy increased apoptosis and decreased proliferation. However, the therapeutic efficacy was satisfactory in the few cases of low CD47 expression. These data indicate that Ara-C, a classical chemotherapeutic drug for acute leukemia treatment, acts synergistically with anti-CD47 monoclonal antibody treatment and that this combination may be an attractive candidate for the therapeutic targeting of AML. 


\title{
Conflicts of interest
}

\author{
The authors declare no conflict of interest.
}

\section{REFERENCES}

Adams GP and Weiner LM (2005). Monoclonal antibody therapy of cancer. Nat. Biotechnol. 23: 1147-1157.

Barclay AN and Brown MH (2006). The SIRP family of receptors and immune regulation. Nat. Rev. Immunol. 6: 457-464. Brown EJ and Frazier WA (2001). Integrin-associated protein (CD47) and its ligands. Trends Cell Biol. 11: 130-135.

Chao MP, Alizadeh AA, Tang C, Myklebust JH, et al. (2010). Anti-CD47 antibody synergizes with rituximab to promote phagocytosis and eradicate non-Hodgkin lymphoma. Cell 142: 699-713.

Chao MP, Alizadeh AA, Tang C, Jan M, et al. (2011). Therapeutic antibody targeting of CD47 eliminates human acute lymphoblastic leukemia. Cancer Res. 71: 1374-1384.

Chiba T, Kita K, Zheng YW, Yokosuka O, et al. (2006). Side population purified from hepatocellular carcinoma cells harbors cancer stem cell-like properties. Hepatology 44: 240-251.

Edris B, Weiskopf K, Volkmer AK, Volkmer JP, et al. (2012). Antibody therapy targeting the CD47 protein is effective in a model of aggressive metastatic leiomyosarcoma. Proc. Natl. Acad. Sci. U. S. A. 109: 6656-6661.

Goodell MA, Brose K, Paradis G, Conner AS, et al. (1996). Isolation and functional properties of murine hematopoietic stem cells that are replicating in vivo. J. Exp. Med. 183: 1797-1806.

Gorman MF, Ji L, Ko RH, Barnette P, et al. (2010). Outcome for children treated for relapsed or refractory acute myelogenous leukemia (rAML): a Therapeutic Advances in Childhood Leukemia (TACL) Consortium study. Pediatr. Blood Cancer 55: 421-429.

Guan Y, Gerhard B and Hogge DE (2003). Detection, isolation, and stimulation of quiescent primitive leukemic progenitor cells from patients with acute myeloid leukemia (AML). Blood 101: 3142-3149.

Guo Y, Follo M, Geiger K, Lübbert M, et al. (2003). Side-population cells from different precursor compartments. $J$. Hematother. Stem Cell Res. 12: 71-82.

Haraguchi N, Utsunomiya T, Inoue H, Tanaka F, et al. (2006). Characterization of a side population of cancer cells from human gastrointestinal system. Stem Cells 24: 506-513.

Heidel F, Solem FK, Breitenbuecher F, Lipka DB, et al. (2006). Clinical resistance to the kinase inhibitor PKC412 in acute myeloid leukemia by mutation of Asn-676 in the FLT3 tyrosine kinase domain. Blood 107: 293-300.

Hosen N, Park CY, Tatsumi N, Oji Y, et al. (2007). CD96 is a leukemic stem cell-specific marker in human acute myeloid leukemia. Proc. Natl. Acad. Sci. U. S. A. 104: 11008-11013.

Hussain SZ, Strom SC, Kirby MR, Burns S, et al. (2005). Side population cells derived from adult human liver generate hepatocyte-like cells in vitro. Dig. Dis. Sci. 50: 1755-1763.

Inaba H, Fan Y, Pounds S, Geiger TL, et al. (2008). Clinical and biologic features and treatment outcome of children with newly diagnosed acute myeloid leukemia and hyperleukocytosis. Cancer 113: 522-529.

Jaiswal S, Jamieson CH, Pang WW, Park CY, et al. (2009). CD47 is upregulated on circulating hematopoietic stem cells and leukemia cells to avoid phagocytosis. Cell 138: 271-285.

Jiang P, Lagenaur CF and Narayanan V (1999). Integrin-associated protein is a ligand for the P84 neural adhesion molecule. J. Biol. Chem. 274: 559-562.

Jin L, Hope KJ, Zhai Q, Smadia-Joffe F, et al. (2006). Targeting of CD44 eradicates human acute myeloid leukemic stem cells. Nat. Med. 12: 1167-1174.

Jordan CT and Guzman ML (2004). Mechanisms controlling pathogenesis and survival of leukemic stem cells. Oncogene 23: 7178-7187.

Jordan CT, Upchurch D, Szilvassy SJ, Guzman ML, et al. (2000). The interleukin-3 receptor alpha chain is a unique marker for human acute myelogenous leukemia stem cells. Leukemia 14: 1777-1784.

Kaspers GJ and Zwaan CM (2007). Pediatric acute myeloid leukemia: towards high-quality cure of all patients. Haematologica 92: 1519-1532.

Kayo H, Yamazaki H, Nishida H, Dang NH, et al. (2007). Stem cell properties and the side population cells as a target for interferon-alpha in adult T-cell leukemia/lymphoma. Biochem. Biophys. Res. Commun. 364: 808-814.

Komuro H, Saihara R, Shinya M, Takita J, et al. (2007). Identification of side population cells (stem-like cell population) in pediatric solid tumor cell lines. J. Pediatr. Surg. 42: 2040-2045.

Lane SW and Gilliland DG (2010). Leukemia stem cells. Semin. Cancer Biol. 20: 71-76.

Lindberg FP, Gresham HD, Schwarz E and Brown EJ (1993). Molecular cloning of integrin-associated protein: an immunoglobulin family member with multiple membrane-spanning domains implicated in alpha $\mathrm{v}$ beta 3 -dependent 
ligand binding. J. Cell Biol. 123: 485-496.

Majeti R, Chao MP, Alizadeh AA, Pang WW, et al. (2009). CD47 is an adverse prognostic factor and therapeutic antibody target on human acute myeloid leukemia stem cells. Cell 138: 286-299.

Matozaki T, Murata Y, Okazawa H and Ohnishi H (2009). Functions and molecular mechanisms of the CD47-SIRPalpha signalling pathway. Trends Cell Biol. 19: 72-80.

Misaghian N, Ligresti G, Steelman LS, Bertrand FE, et al. (2009). Targeting the leukemic stem cell: the Holy Grail of leukemia therapy. Leukemia 23: 25-42.

Monaco G, Konopleva M, Munsell M, Leysath C, et al. (2004). Engraftment of acute myeloid leukemia in NOD/SCID mice is independent of CXCR4 and predicts poor patient survival. Stem Cells 22: 188-201.

Ravandi F and Estrov Z (2006). Eradication of leukemia stem cells as a new goal of therapy in leukemia. Clin. Cancer Res. 12: 340-344.

Setoguchi T, Taga T and Kondo T (2004). Cancer stem cells persist in many cancer cell lines. Cell Cycle 3: 414-415.

Testa U (2011). Leukemia stem cells. Ann. Hematol. 90: 245-271.

Tsuchiya S, Yamabe M, Yamaguchi Y, Kobayashi Y, et al. (1980). Establishment and characterization of a human acute monocytic leukemia cell line (THP-1). Int. J. Cancer 26: 171-176.

van Rhenen A, van Dongen GA, Kelder A, Rombuouts EJ, et al. (2007). The novel AML stem cell associated antigen CLL-1 aids in discrimination between normal and leukemic stem cells. Blood 110: 2659-2666.

Wang JC and Dick JE (2005). Cancer stem cells: lessons from leukemia. Trends Cell Biol. 15: 494-501.

Woods WG (2006). Curing childhood acute myeloid leukemia (AML) at the half-way point: promises to keep and miles to go before we sleep. Pediatr. Blood Cancer 46: 565-569.

Zhou S, Morriss JJ, Barnes Y, Lan L, et al. (2002). Bcrp1 gene expression is required for normal numbers of side population stem cells in mice, and confers relative protection to mitoxantrone in hematopoietic cells in vivo. Proc. Natl. Acad. Sci. U. S. A. 99: 12339-12344. 